\title{
LA JUNTA DE ADELANTO DE ARICA Y JOHN V. MURRA. DOS LECTURAS SOBRE EL DESARROLLO ANDINO EN EL NORTE DE CHILE
}

\author{
THE JUNTA DE ADELANTO DE ARICA AND JOHN V. MURRA. \\ TWO READINGS ON THE DEVELOPMENT OF THE ANDEAN POPULATION \\ OF NORTHERN CHILE
}

\author{
Luis A. Galdames Rosas ${ }^{1}$ y Rodrigo D. Ruz Zagal ${ }^{1}$
}

\begin{abstract}
Este trabajo contextualiza y analiza la intervención llevada a cabo el 23 de julio de 1975 por el Dr. John V. Murra en el marco de una reunión destinada a discutir estrategias de desarrollo que la Junta de Adelanto de Arica [en adelante J.A.A.] trataba de impulsar para los sectores rurales de Arica. Este encuentro, realizado en la sede de este organismo no-gubernamental, fue promovido por el Departamento de Antropología de la Universidad del Norte, con miras a darle una orientación más antropológica a las propuestas desarrollistas, por lo que se vieron confrontadas dos posiciones en torno a esta materia. A continuación de nuestro análisis se transcribe el texto completo de dicha reunión que muestra las posiciones vigentes en dicha época.
\end{abstract}

Palabras claves: Junta de Adelanto de Arica, John V. Murra, etnohistoria andina, desarrollo.

This paper conceptualizes and analyzes the presentation of Dr. John V. Murra in a meeting intended to discuss strategies for the development of rural sectors of Arica, organized by Junta de Adelanto de Arica (JAA) on July 23, 1975. The meeting, which took place in the headquarters of this non-governmental organization, was arranged by the Departamento de Antropología of the Universidad del Norte with the intention of giving an anthropological orientation to the development program. Participants confronted two positions on this matter. Following our analysis, the complete text of the meeting is transcribed, which shows the current positions of that epoch.

Key words: Junta de Adelanto de Arica, John V. Murra, Andean ethnohistory, development.

El propósito del presente trabajo consiste en realizar una lectura contextualizada del documento de la J.A.A. "Visita al Comité del Señor John Murra quien realiza trabajos antropológicos en la zona altiplánica, de fecha 23 de Julio de 1975" $(\text { Anexo) })^{2}$.

Para ello entregaremos algunos antecedentes en torno a la J.A.A, para luego examinar brevemente el estado del arte de las disciplinas sociales practicadas en el extremo Norte de Chile en relación a "lo andino" y la influencia que ejerció en los investigadores locales el pensamiento orientador de John V. Murra, a mitad de los años setenta.

Ya en relación evidente con el documento al que hemos hecho mención, procuraremos establecer lo que nos parece la confrontación entre dos concepciones del mundo: la desarrollista representada institucionalmente por la J.A.A. y la mirada etnológica encarnada en los dichos del propio Murra.

\section{El Estado Chileno y la Junta de Adelanto de Arica}

En regiones fronterizas incorporadas vía conflicto bélico con un país vecino, con características demográficas y étnicas tan diversas, como es el caso de Arica (Galdames et al. 1981), la acción del Estado se encuentra cruzada por racionalidades múltiples

que impiden dar coherencia, armonía y complementariedad al desarrollo. La región como espacio en que habita la sociedad civil absorbe estas racionalidades [constituyendo] una deformación en el modelo de desarrollo capitalista (Podestá 2004:102).

En el marco de la vigencia del modelo del Estadocompromiso, el Presidente Carlos Ibáñez del Campo en representación del Estado chileno creó en 1953 el denominado "Puerto Libre", buscando a través

1 Departamento de Ciencias Históricas y Geográficas, Universidad de Tarapacá, Avenida 18 de Septiembre 2222, Arica, Chile. lgaldame@uta.cl; rruz@uta.cl 
de este mecanismo revertir la situación de atraso económico que vivía la ciudad a través de políticas de excepción que favorecieran su desarrollo. A este primer instrumento le siguió otro diseñado por el mismo mandatario pero que fue llevado a cabo por el Gobierno de Jorge Alessandri Rodríguez a partir de 1958. Para ello se crea la J.A.A., que con autonomía en lo que decía relación con el patrimonio, los presupuestos y las decisiones, pretendía erigirse en el modo válido de regionalización, favoreciendo el desarrollo de la industria y convirtiéndose en el motor de la inversión pública.

Pese a lo obrado, el impacto de este último instrumento no logró mayor efecto, perdiéndose así la idea de originar un círculo virtuoso que rompiere las estructuras de la pobreza (Galdames 1978).

En complemento a la implementación de obras y políticas de desarrollo en el sector urbano, la intención de producir desarrollo en el hinterland ariqueño comenzó a manifestarse a partir de la década de 1960, momento en el que se realizan diagnósticos y estudios exploratorios llevados a cabo por especialistas de las ciencias sociales en el sector andino, coordinando esfuerzos con organismos internacionales como el Programa de las Naciones Unidas para el Desarrollo [PNUD] y la Organización Internacional del Trabajo [OIT], que se materializaron en una suerte de estrategia de desarrollo dirigida a las comunidades y que dio nacimiento al Plan Andino. De este modo, se pensaba que a los aspectos productivos debía adicionarse lo que en la época se conoció como "dimensión social del desarrollo"3.

Esta nueva preocupación de la J.A.A. se dio además en un contexto teórico en el que corrientes referidas al cambio social, inspiradas en el social cristianismo y el marxismo, creyeron ver en la población andina potencialidades sociales y colectivas propicias para una sociedad socialista (Gundermann y González 2009; Núñez 1967; Rivera 1972-1973).

El Plan Andino gestionó, para la zona de Arica, en coordinación con otras instituciones públicas, recursos importantes para obras de infraestructura y equipamiento urbano (agua potable, alcantarillado, baños públicos, sedes sociales, luz eléctrica), de conectividad (redes viales), de mejoramiento de condiciones productivas y tecnológicas (canalización, equipamiento). No resulta nítido, sin embargo, que el otorgamiento de estos recursos haya obedecido a una política pública consistente o si, simplemente, destinó recursos a medida que le eran solicitados por los pobladores del interior de Arica.

Con la ruptura del orden institucional vigente y el advenimiento del régimen militar, a partir de 1974 se crea la Comisión de Desarrollo del Interior como parte del Plan Andino. Aquí se continuó la inversión en infraestructura y en programas asistenciales, pero ahora de la mano de una visión geopolítica que mostraba preocupación por el espacio andino en su condición de espacio "limítrofe" con dos países considerados potencialmente hostiles.

Desde la perspectiva de los pueblos del interior de la provincia, la existencia de la J.A.A. supuso de un modo u otro recuperar vínculos de participación y de interacción con el Estado que habían estado adormecidos durante décadas anteriores ${ }^{4}$.

\section{Las Ciencias Sociales hacia Mediados de 1970 en Arica}

En el contexto de las Ciencias Sociales la disciplina que destacó en la década de 1970 en cantidad y calidad de estudios referidos a Arica fue la arqueología desarrollada en las sedes universitarias instaladas en la ciudad 5 .

Un espacio más limitado pero no menos calificado lo cubrió la antropología cultural, con la presencia de Tristan Platt y las influencias cercanas de Gabriel Martínez desde Isluga. La Etnohistoria, en tanto, quedó en manos del único cultor de la época: Jorge Hidalgo, ligado fuertemente a John V. Murra.

Acaso sea menester señalar que el rol articulador de estos saberes disciplinarios, emparentados pero diferentes, quedó por entonces bajo la responsabilidad de los cultores de las disciplinas sociales en las sedes universitarias regionales, en donde se advirtió que el conocimiento de la arqueología resultaba indispensable para el antropólogo y para el etnohistoriador y viceversa considerando que el conocimiento de estas últimas disciplinas podía ser muy revelador para alcanzar lecturas arqueológicas distintas. Acaso sin saberlo se comenzaba a llevar a cabo en Arica la interdisciplinariedad que había proclamado la escuela de los Annales ${ }^{6}$.

En especial cabe reconocer en el doctor John V. Murra su rol facilitador de este proceso de trabajo en común, cuya tesis fundamental sobre los Andes ya había sido difundida en Arica. A ello deben agregarse las permanentes visitas que el Dr. Murra comenzó a realizar a la ciudad de Arica, 
lo que fue creando lazos teóricos y personales que surgieron y se fueron haciendo más estrechos con el transcurrir del tiempo ${ }^{7}$.

Más o menos en esta época, comienza a utilizarse la noción de "lo andino" en el Norte de Chile, como concepto genérico que hace alusión explícita a grupos étnicos coloniales y prehispánicos asentados en el territorio, produciéndose un manejo de conceptos que años después se harán comunes a todas las disciplinas sociales y humanistas ${ }^{8}$.

Estas ideas fueron recogidas tempranamente por el Departamento de Antropología de la Universidad del Norte y la J.A.A., otorgando un espacio de discusión para las temáticas andinas, en donde la experiencia de John V. Murra venía a fortalecer el interés de ambas instituciones por comprender las dinámicas culturales, sociales y económicas de la población andina ariqueña.

\section{Análisis del Documento "Visita al Comité del Señor John Murra quien realiza trabajos Antropológicos en la Zona Altiplánica" (23 de Junio de 1975)}

La materialización de la reunión realizada el 23 de junio de 1975 habría sido el fruto de una idea promovida por los investigadores del Departamento de Antropología de la Universidad del Norte en acuerdo con la J.A.A., lo que permitió la existencia de un acta documental que pasamos a comentar a continuación.

El Acta $N^{\circ} 11 / 75$ de la Comisión de Desarrollo del Interior de la J.A.A. da cuenta de lo expuesto por diferentes participantes en la reunión con el Dr. Murra, que asiste como invitado.

Para efectos metodológicos utilizaremos de manera comparativa los razonamientos discursivos expuestos inicialmente por el presidente de la comisión, Sr. Jorge Vallejo Arcos, en representación de la J.A.A. y, a continuación, se señalan resumidamente los comentarios del Dr. John V. Murra. A partir de éstos elaboraremos un cuadro de oposiciones no complementarias, en donde se identifican ideas y temas centrales, los que se problematizan, y se le otorgan eventuales soluciones, concluyendo con los supuestos que subyacen tras los razonamientos de ambas partes.

Las ideas centrales giran en torno a aspectos demográficos, culturales y tecnológico-productivos que permiten visualizar con mayor nitidez las miradas contrapuestas manifiestas al dar cuenta de la situación de los pueblos del interior de Arica

En lo sustancial, se presenta el diagnóstico institucional de la J.A.A. expresado por Vallejo Arcos, al cual se contrapone la visión etnológica representada por John V. Murra.

I. Aspectos Demográficos

\begin{tabular}{l}
\hline \\
\hline Junta de Adelanto de Arica \\
\hline Problema: \\
* La progresiva migración rural-urbana, que se manifiesta en el \\
abandono de población de sus pueblos de origen para asentarse \\
en las zonas de desarrollo urbano, en este caso representadas \\
por la ciudad de Arica.
\end{tabular}

\begin{abstract}
Solución:
* Reproducir en los pueblos el modo de vida urbano que supuestamente satisface la ciudad de Arica (v. gr. habilitación de servicios médicos, infraestructura urbana y vial, etc.), pensando que de esta manera se haría innecesario el desplazamiento de la población hacia la ciudad-puerto.
\end{abstract}

\section{Supuesto:}

* La noción del "interior" representa un grado inferior de desarrollo frente a la ciudad, constituyendo ambos un continuo cultural, económico y social que debe ser recorrido inevitablemente. Desde una visión urbana occidental, lo "distinto" (lo andino) es considerado como atraso.

\section{Dr. John V. Murra}

Problema:

* Relativiza el problema identificado por la institución (J.A.A.), a partir del cuestionamiento de la idea de migración que conduciría a un abandono de las localidades de origen.

Señala la existencia de estructuras históricas de movilidad poblacional andina y su capacidad de respuesta a políticas estatales.

\section{Solución:}

* Que la J.A.A., en representación del Estado Nación, asuma que los movimientos poblacionales no representan problemas ni novedad desde la perspectiva de la población nativa.

\section{Supuesto:}

* Supuesto etnológico que destaca la visión étnica de movilidad y de uso de múltiples espacios, en oposición a la visión estatal occidental que asocia la migración con desarraigo. 
II. Aspectos Culturales

Choque Cultural

\begin{tabular}{l} 
Junta de Adelanto de Arica \\
\hline Problema: \\
* Del proceso migratorio señalado más arriba, se desprenderían \\
conflictos para el migrante andino al experimentar éste menoscabo \\
frente a procesos de adaptación a una cultura urbana y nacional \\
distinta y dominante.
\end{tabular}
distinta y dominante.

\section{Solución:}

* Reafirmar propuestas de arraigo territorial y reproducir modelos productivos y de infraestructura urbana en las localidades interiores.

Generar instancias propositivas para minimizar el impacto o "choque cultural" a partir de diagnósticos e investigación aplicada generada por cientistas sociales.

\section{Supuesto:}

* A partir de propuestas externas a las concepciones originarias, reproducir modelos y realidades urbanas que generen el arraigo de la población andina a sus pueblos de origen, lo que evitaría la migración y los conflictos que ello provoca.

\section{Dr. John V. Murra}

\section{Problema:}

* Desde la óptica etnológica, el problema del "choque cultural" experimentado por el andino se invierte. En realidad, el conflicto lo viven las entidades receptoras de migrantes (la ciudad y el poblador urbano).

El "problema" se revierte y se ubica en la urbe y no en la población andina, producto de las estructuras urbanas que tradicionalmente han tendido a desplazar y marginar a aquellos que no responden a sus lógicas citadinas.

\section{Solución:}

* Destacar el rol activo de la dinámica poblacional indígena en procesos económicos y políticos profundamente históricos, que han arrancado de la necesidad del hombre andino por adaptarse eficientemente en escenarios históricos cambiantes. Para ello se hace necesario oír sus experiencias y estar atento a sus propuestas.

\section{Supuesto:}

* La población andina no ha sido ajena a contactos con una cultura y sociedad "dominante". En cambio la sociedad y cultura chilena no tiene registro de cómo responder al "otro", el mundo cultural andino.

\section{Tecnología y Producción}

Modernización tecnológica y mejoras productivas

\begin{tabular}{l} 
Junta de Adelanto de Arica \\
\hline Problema: \\
* Las tecnologías tradicionales son "anticuadas", manteniendo \\
un "atraso" tecnológico traducido en una baja producción y \\
productividad.
\end{tabular}

Solución:

* Introducir y modernizar a partir de nuevas tecnologías conducentes a revertir las condiciones de "atraso" y escasa productividad.

\section{Supuesto:}

* El salto del subdesarrollo agrícola al desarrollo sólo se logra a partir de la introducción y producción sustentada en tecnología moderna.

\section{Dr. John V. Murra}

\section{Problema:}

* La problemática pasa por estructuras históricas "recientes" derivadas de cambios en los medios de uso y acceso a la propiedad de la tierra, así como al arrinconamiento a asentamientos mínimos que explican la baja productividad y la alta migración de población indígena.

\section{Solución:}

* Destacar y considerar en políticas de la materia, elementos distintivos de la economía andina tales como manejo de espacios, pisos ecológicos y microclima en función de la productividad.

\section{Supuesto:}

* Un manejo exitoso en materia productiva por parte de la población andina coherente con el manejo tradicional de su tecnología, espacio, clima y procesos productivos.
Del análisis de los razonamientos revisados, de parte de la J.A.A. es posible reconocer una visión desarrollista que mira a los pueblos del interior como entidades subdesarrolladas, en estado de pobreza o de carencia. Enfatiza la mirada económica y social para desde allí promover desde "arriba" y desde "fuera" una suerte de impulso civilizador que eleve las condiciones de vida de la población dentro de una sociedad que se concibe como homogénea y que, por lo mismo, no otorga mayor relevancia a la cultura e historia de las poblaciones del interior de Arica.

De otro lado, una mirada etnológica argumenta que lo que está en pugna en realidad son dos lógicas culturales diferentes, y por ende otorgan significados distintos a los fenómenos naturales, 
sociales y económicos. Con un discurso simple y pedagógico, John Murra busca instalar la mirada del "otro", del supuestamente inferior, colocando en entredicho el supuesto que todas las sociedades y todas las culturas se comportan de forma similar y buscan los mismos fines.

Agradecimientos: El presente trabajo se incluye dentro de los resultados del proyecto FONDECYT 1095130 "Las ideas para encarar la crisis y el desarrollo endógeno en el Norte Grande, 1950-1966.
Política, economía y cultura". Nuestros agradecimientos al equipo de trabajo del Archivo Histórico Vicente Dagnino en especial al asistente Sr. Daniel Castillo y al equipo de ayudantes, alumnos de Licenciatura en Historia Sres. Renato Calderón, Diego Quiroz y Carlos Toloza quienes realizaron la transcripción del escrito que se adjunta; finalmente, los autores desean agradecer las sugerencias de los evaluadores y editores hacia nuestro manuscrito cuyo aporte mejoró la versión original permitiendo su publicación.

\section{Referencias Citadas}

Castro, V., C. Aldunate y J. Hidalgo, editores 2000 Nispa Ninchis/Decimos Diciendo: Conversaciones con John Murra. Instituto de Estudios Peruanos (I.E.P.), e Institute of Andean Research (I.A.R.), Lima.

Galdames, L.

1978 Notas Epistemológicas sobre la Teoría de la Dependencia. Instituto Latinoamericano de Doctrina y Estudios Sociales (I.L.A.D.E.S), Santiago.

Galdames, L., W. Ríos, P. Dauelsberg, S. Chacón y L. Alvarez 1981 Historia de Arica. Ilustre Municipalidad de Arica, Editorial Renacimiento, Santiago.

Gundermann, H. y H. González 2009 Sociedades indígenas y conocimiento antropológico. Aymaras y atacameños del Norte de Chile. Chungara Revista de Antropología Chilena 41:113-164.

Lara, R.

1972-1973 Un año más del Museo Arqueológico San Miguel de Azapa. Chungara 1-2:7-9.

Núñez, L.

1967 Arqueología y Universidad. Ancora 3:119-126. Antofagasta. 2006 Testimonios en torno a la construcción de un ideario de integración arqueológica para el Centro Sur Andino, en Esferas de Interacción Prehistóricas y Fronteras Nacionales Modernas: Los Andes Sur Centrales, editado por H. Lechtman, pp. 621-630. Instituto de Estudios Peruanos (I.E.P.), Lima e Institute of Andean Research (I.A.R.), New York.
Murra, J.

1962 An archaeological "restudy" of an Andean ethnohistorical account. American Antiquity 28:1-4.

1972 El "control vertical" de un máximo de pisos ecológicos en la economía de las sociedades andinas. En Visita de la Provincia de León de Huánuco en 1562, editado por J.V. Murra, vol. 2, pp. 429-476. Universidad Hermilio Valdizán, Huanuco.

Murra, J. y M. López-Baralt, editores

1998 Las Cartas de Arguedas. Fondo editorial Universidad Católica del Perú, Lima.

Orellana, M.

1996 Historia de la Arqueología en Chile. Colección de Ciencias Sociales Bravo y Allende editores, Santiago.

Podestá, J.

2004 La Invención de Tarapacá. Estado y Desarrollo Regional en Chile. Iquique. Ediciones Campus, Universidad Arturo Prat, Iquique.

Rivera, M.

1972-1973 A manera de introducción; hacia una política de desarrollo integral del Departamento de Arica. Chungara 1-2:11-16.

Salomon, F.

2010 Murra en la selva de paja. Chungara Revista de Antropología Chilena 42:13-18.
1 Nota de los Editores: En más de una oportunidad John Murra nos comentó sobre esa reunión a la que le atribuyó una importancia histórica vital y quedó más interesado aún cuando le señalamos que existía una transcripción de las grabaciones, lo que nunca llegó a ver. Copias de este documento existían en la Universidad de Tarapacá a mitad de los años setenta. Hace pocos meses Rodrigo Ruz me envió copia de una trascripción digital del documento original, que decidimos publicar junto con el análisis histórico cultural que entregan Luis Galdames y Rodrigo Ruz.

2 Documento recientemente localizado en dependencias del Archivo Histórico Vicente Dagnino de la Universidad de Tarapacá, unidad en la que se resguarda el material producido durante los años 1958 y 1976 perteneciente a la desaparecida J.A.A. Uno de estos fondos documentales, "Estudios Técnicos de la J.A.A.", contiene información relevante al denominado "Plan Andino". Entre las piezas documentales que contiene dicho fondo se localizó el documento que presenta como addenda del presente artículo. El registro original pudo haberse soportado en cinta magnetofónica, la cual fue mecanografiada en formato de acta por miembros de la J.A.A. y depositada en sus archivos hacia la década de 1970. En la actualidad y bajo la realización del seminario "Fuentes documentales para la historia regional" dictada por el Dr. Luis Galdames en coordinación con el archivo, se identificó y transcribió nuevamente el original que ahora se presenta y divulga. 
3 John Murra habría tenido experiencia tangencial en programas de desarrollo en sectores andinos en momentos previos a su ingreso a la Universidad de Cornell, ello se desprende de correspondencia intercambiada con José María Arguedas en donde este solicitaba la realización de informes referentes al denominado proyecto Perú Cornell impulsado por dicha Universidad en Vicos (Ancash, Perú) en 1952 (Murra y López-Baralt 1998). El proyecto buscaba el generar cambios inducidos en la población indígena a partir de la integración al sector moderno occidental. En este proyecto habrían participado importantes antropólogos tanto peruanos como extranjeros, y habría sido un referente importante en la posición distante de Murra frente a proyectos de carácter desarrollista, y por ende en relación a la antropología aplicada que cobra fuerza a partir del término de II Guerra Mundial. Este punto aparece reafirmado en Salomon (2010), en este número.

4 Esta forma de vinculación ha incentivado en el presente a investigar más y de modo compartido con otras disciplinas sociales los mecanismos que la población andina sostuvo con los Estados republicanos (tanto el chileno como el peruano).

5 A la sazón, en Arica existían dos sedes universitarias: la de la Universidad de Chile y la de la Universidad del Norte, cuyos académicos e infraestructura conformarían lo que a partir de 1982 será la Universidad de Tarapacá. En la década de 1970 en la Universidad de Chile se desempeñaban en la línea de investigación arqueológica Luis Álvarez, Percy Dauelsberg y Sergio Chacón, en tanto en la Universidad del Norte trabajaban en la disciplina Guillermo Focacci y los arqueólogos de profesión Mario Rivera y Gonzalo Ampuero, además de otros profesionales vinculados a la investigación arqueológica, en especial al ámbito de la museología.

6 Es altamente probable que la antropología haya sido, junto al cultivo de las demás ciencias sociales y las humanidades el área de conocimiento que sufrió mayormente el impacto del Golpe Militar de 1973 que asociaba estos saberes a corrientes ideológicas de carácter "subversivo". La arqueología aparentemente no despertó los mismos temores y de algún modo la llegada del nuevo régimen no afectó significativamente el avance de la disciplina. Mayores detalles y antecedentes en Gundermann y González (2009).

7 Sobre una mirada actual orientada a la evaluación del aporte de Murra en las distintas vertientes de las ciencias sociales en Chile y el norte grande ver Orellana (1996), Núñez (2006) y Gundermann y González (2009). Un enfoque más íntimo y relacionado a aspectos biográficos, así como a la construcción de vínculos personales y académicos ver Castro et al. (2000).

8 Es a inicios de la década de 1970 donde la obra de John V. Murra adquiere un carácter de modelo explicativo a partir de la difusión de su obra sobre el control vertical de un máximo de pisos ecológicos en Huánuco, ver Murra (1972). Posteriormente, el concepto "andino" comienza a hacerse extensivo a la comunidad científica.

Nota de los editores: Murra anticipó en el año 1962 la necesidad de colaboración entre arqueólogos, etnólogos, etnohistoriadores, sobre lo que insiste en 1972. 


\title{
ANEXO \\ DOCUMENTO ${ }^{1}$ \\ VISITA AL COMITÉ DEL SR. JOHN MURRA QUIEN REALIZA TRABAJOS ANTROPOLÓGICOS EN LA ZONA ALTIPLANICA.
}

\author{
Junta de Adelanto de Arica \\ Ley $\mathbf{N}^{\circ} 13.039$

\section{Comisión de Desarrollo del Interior Acta $\mathbf{N}^{\circ}$ 11/75}

En Arica, a 23 de Julio de 1975, entre las 15,45 y 17,45 se reunió en la Sala de Sesiones de la Institución del Comité de Desarrollo del Interior, bajo la presidencia del titular señor Jorge Vallejos Arcos y contando con la asistencia de las siguientes personas:
D. John Murra
D. Carlos Solari H.
D. Arturo Solar G.
D. Mario Rivera
D. Maria Elena Schönherr
D. Rafael Montes G.
D. Eugenio Doussoulin
D. Karin Von Buch
D. Héctor Parada
D. Liliana Ulloa
D. Jorge Hidalgo
D. Virginia Popper
D. José Weimborn
D. Eugenio Sotomayor
D. Julia Cándova [Córdova]
D. Donald Erskine
D. Sergio Erices
D. Gabriel Martínez
D. Tristan Platt
D. Brigitte de Gonneville

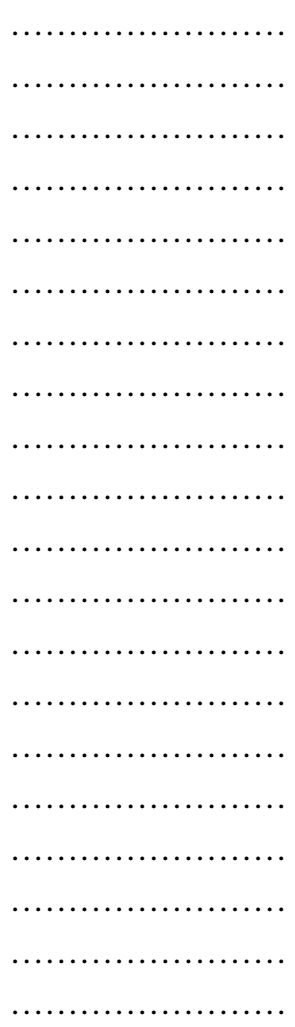

Doctor- Antropología

Plan Andino JAA

Oficial Enlace - Educación

Depto. Antrop. U. del Norte

ODEPA

CORFO

CORA

MINVU

INACAP

Depto. Antrop. U. del Norte

Depto. Antrop. U. del Norte

Depto. Antrop. U. del Norte

Depto. Agricult. U. del Norte

Depto. Agricult. U. del Norte

Depto. Antrop. U. del Norte

SAG

Depto. Antrop. U. del Norte

Centro Isluga U. del Norte Iquique

Depto. Antrop. U. del Norte

Depto. Antrop. U. del Norte

\footnotetext{
Se ha respetado la sintaxis del documento original, pese a los ripios de estilo y forma que esta presenta. En casos especiales, se ha modificado la ortografía original, como por ejemplo en el caso de tildes de palabras. Además se ha utilizado la locución [sic] para señalar la representación textual del documento transcrito, de la misma manera se ha utilizado la frase [sin transcripción] para señalar la falta de términos que la transcripción pasó por alto y que se expresan en puntos suspensivos en el documento expuesto.
} 


\section{Gonzalo Ampuero \\ D. Flavio Piazza \\ D. Patricia Soto \\ D. Rolando Cisternas \\ D. Domingo Aranguiz \\ D. Miguel Fuertes \\ D. Fernando Graña \\ D. Dagoberto Cifras \\ D. Rubén Campos \\ D. Orlando Saavedra \\ D. Nancy Alanoca \\ D. Enrique Araneda \\ D. Luciano Arévalo \\ D. Freda Quinteros R.}

Depto. Antrop. U. del Norte

Depto. Antrop. U. del Norte

Depto. Antrop. U. del Norte

INDAP

INDAP

Depto. Agricult. U. del Norte

Depto. Agricult. U. del Norte

SAG

Director Escuela N ${ }^{\circ} 17$ Putre

Bienes Nacionales

CORA

SERCOTEC

Vialidad

Secretaría Comisiones de JAA
SR. VALLEJO: Se inicia la sesión extraordinaria del Comité de Desarrollo del Interior y el señor Mario Rivera tendrá a su cargo la presentación del Antropólogo señor John Murra.

SR. RIVERA: No deseo hacer una gran introducción en la presentación del señor Murra, ya que sus actividades son bastante conocidas. Solamente deseo decir que viene hasta Arica en una misión de esfuerzo personal, acaba de terminar una serie de conferencias auspiciadas por el Departamento de Estado de los Estados Unidos y al término de estas sesiones -a todo tipo de público- ha querido llegar hasta esta región con motivaciones de tipo más personal, y de paso, para desarrollar sus propias investigaciones en Bolivia, específicamente en Sucre.

El Dr. Murra, además, ha trabajado en la región Andina durante años y el conocimiento que tiene y más que nada la experiencia, creemos que es invaluable desde este punto de vista y es ese trabajo el que queremos compartir con todos ustedes de una u otra manera y yo preferiría que sea desde un punto de vista más informal cosa que se puedan entregar más informaciones, tanto de él como la que puedan dar ustedes en cuanto a los programas que están desarrollando en el Interior.

SR. VALLEJO: Yo creo que la mejor forma de abordar la situación es hacer una pequeña introducción de lo que es el Comité de Desarrollo del Interior y posteriormente hacer una mesa redonda donde los participantes puedan hacer consultas o puedan plantear puntos de vistas.

El Comité de Desarrollo de Interior está funcionando desde Marzo del año pasado por disposición de la autoridad departamental de Arica, que le dio vida a través de un bando y en el cual incorporó a todas las Instituciones que, de una u otra manera, tienen que ver algo con el Interior.

La razón fundamental que llevó a la autoridad a crear este comité, fue que -en rigor de verdad- a pesar del gran desarrollo que se había producido aquí en la zona, el desarrollo práctico y físico se había concentrado en la ciudad de Arica. Naturalmente que esto produjo una atracción extraordinariamente grande que aceleró el proceso de migración que se estaba produciendo desde el Interior hacia la ciudad desde hace bastantes años.

Preocupados por esta situación, la autoridad creó este comité con el objeto que definiera alguna línea y algunos planes que pudieran permitir -a un mediano plazo o a un largo plazo- tratar de consolidar un desarrollo en el Interior, tomando en cuenta las características básicas físicas y las humanas, ya que es esta una de las pocas zonas del país en la cual hay un contingente de personas nativas que tienen características diferentes que el resto del país (cultural), o la que predomina en el resto del país.

El Comité empezó a funcionar desde el mes de Abril del año pasado, con el objetivo de poder establecer un programa de acción. Durante el año pasado y hasta Marzo de este año se estuvo 
reuniendo en cuyo mes de dio fin a la primera parte de su actividad con la publicación de un trabajo que se llama "Plan Quinquenal de Inversiones para el Interior del Departamento de Arica", el cual fue enviado al Departamento de Antropología de la Universidad del Norte.

En este libro hay un diagnóstico de la situación, hay un análisis de las posibilidades que se tienen y se estableció un programa quinquenal de inversiones, que en este momento está sometido a la consideración de la autoridad y esperamos la resolución de ella para saber de qué se dispone, ya que evidentemente todos estos problemas en gran medida, se resuelven por la vía financiera, ya que si no hay financiamiento difícilmente se puede solucionar algo.

Hay que hacer notar que esto no es nuevo en el Departamento de Arica. Poco después de la creación de la Junta de Adelanto, por el año 60-61, la Junta tomó contactos con las Naciones Unidas, especialmente con la OIT y se hizo un convenio de asesoría, por lo cual llegaron algunos expertos de OIT, de UNESCO y del PNUD que estuvieron trabajando durante algunos años. Concretamente OIT, que es especialista en Plan Andino, estuvo trabajando durante unos 6 años y con lo cual se iniciaron las actividades de promoción social en el área del Interior, con el objetivo fundamental de poder iniciar algunas obras de tipo comunitario que vendrían a satisfacer las necesidades -más bien-inmediatas que se presentaban.

Desgraciadamente en esto no hubo un plan específico, concreto, sino que se fue realizando al andar y se fueron resolviendo los problemas que se presentaban en forma inmediata. De esto hay una serie de realizaciones concretas como es el caso del Departamento de Plan Andino, que la Junta creó y que todavía mantiene el cual está orientado fundamentalmente al mejoramiento de la situación de la gente del Interior y a la realización de obras, tal es así que se han creado varias policlínicas en diversas localidades del Interior, se ha mejorado la situación caminera y se han hecho algunas obras de infraestructura, mas que nada con la agricultura en lo que se refiere a regadío. Todo esto se ha hecho en pequeña escala, con la ayuda comunitaria fundamentalmente y la ayuda financiera de la Junta de Adelanto que permanentemente ha estado manteniendo esto.

En el análisis que se hizo aquí en el Comité en cierta medida se hizo una evaluación de lo realizado y a raíz de eso se confeccionó el plan quinquenal de inversiones.

Esto es lo que se podría decir de lo que ha estado sucediendo acá. Ahora, desde el punto de vista sociológico, evidentemente que hemos tenido bastantes problemas; hay problemas de migración, tal como lo habíamos manifestado. Yo creo que todos los aquí presentes y que han tenido contactos con la gente, están conscientes que han tenido problemas de choque cultural e incluso en la ciudad misma hay un problema que puede agravarse por la situación cultural, de migrantes que se han incorporado a una cultura diferente, como es la cultura urbana, con toda la secuela de problemas que ellos traen.

Lo que más nos preocupa a nosotros es el aspecto de abandono que se está produciendo en el Interior por parte de los nativos, que a través del desarrollo de la ciudad, y que en cierta medida y un poco paradójicamente, facilitado por las obras de infraestructura que se han realizado, se vienen a la ciudad en bus, micro o auto, en vez de venirse en mula como se hacía antiguamente.

De todas maneras creo que algo se ha hecho, el problema que se tiene es fundamentalmente de ajuste.

Eso sería a grandes rasgos. No sé si alguno de los presentes deseen hacer algún alcance. Rogaría a las personas que hagan uso de la palabra, lo hagan en forma breve y precisa para ganar tiempo.

Yo estimo que quizás es una cosa importante y que habría que destacar, es la preocupación que ha habido en el seno del Comité, especialmente en lo que se refiere al aspecto de investigación para el desarrollo. En rigor de verdad, debemos establecer que en la reunión pasada se planteó un debate bastante fuerte sobre cómo ahorrar todo este problema de tenencia de la tierra y algunos aspectos de introducción de la tecnología con aprovechamiento de la tecnología particular de los nativos.

Los que están presentes han tenido oportunidad de tomar contacto estrecho con la gente y se han dado cuenta evidentemente, que si bien los sistemas tradicionales de explotación de la tierra o explotación ganadera que son los rubros fundamentales que maneja esta gente, son evidentemente anticuados porque son los tradicionales. Se ha podido comprobar que hay muchas cosas también que son de valor y han tenido en el Comité el reconocimiento de parte de algunos profesionales de que hay algunas cosas que desde el punto de vista de la tecnología actual, realmente no se superan está el caso concreto del 
Algodonal en el cual el nativo con mucha habilidad ha salvado el problema de.......de [sin transcripción.] pequeña escala con lo que significa transportar agua desde una zona desértica con tierras absorbentes y todo lo demás.

La inquietud de los miembros del Comité es que no tenemos el cabal conocimiento de cuáles son las formas operatorias de esta gente como para poder evaluarlas y sacarles el máximo de provecho de ello, o mejorarlas sin hacer grandes modificaciones, de tal manera de poder adecuar la productividad que ellos tienen en este momento -que es baja- a una mejor productividad sin la introducción radical del sistema y de técnicas realmente nuevas.

El Departamento de Antropología nos hizo una proposición, la cual la tenemos en consideración pero creemos que hay que afinar bastante este asunto, no porque tengamos desconfianza de la forma como se vaya a lograr la investigación, sino que a nosotros nos preocupa mas que la investigación en si, como es el caso de antropología que trabaja fundamentalmente investigando, es la investigación para poder sacar provecho desde el punto de vista del desarrollo comunal.

Creemos que Usted, en este sentido, pueda darnos alguna orientación ya que debemos reconocer hidalgamente que a veces no podemos resolver este problema y para nosotros es un callejón sin salida.

Fundamentalmente abordarlo a bajo costo, con soluciones concretas relativas a corto plazo, ya que naturalmente las autoridades cuando uno les plantea una investigación que se va a demorar unos 2, 3, 4 o 5 años, se ponen muy escépticos, se dan, muchas soluciones pero el proyecto concreto del estudio no se ve.

Sigue ofrecida la palabra.

SRA. NANCY ALANOCA: Yo pienso que es mejor que el Dr. Murra nos hiciera una exposición de los aspectos fundamentales de la cultura andina y de eso hacer las consultas.

DR. MURRA: Deseo empezar mi exposición manifestando que es solo la tercera vez que estoy en esta zona y mis conocimientos, como lo dijo el señor Rivera, han sido foráneos, mi interés ha sido siempre trabajar en bien del hombre andino y saber lo que el hombre andino ha investigado por su cuenta y para sí. Como digo es solo la tercera vez que visito el Norte Grande, por lo tanto mis conocimientos son muy limitados.
Nosotros tuvimos una larga sesión en la Universidad y uno de los jóvenes manifestó si yo no había tomado en cuenta algunas de las sugerencias que había hecho, pues eso implicaría un choque cultural a la inversa, no es el choque del hombre que se viene a la ciudad y que tiene que adaptarse a las condiciones tan diferentes y esto no solo sucede en esta ciudad sino que en todas partes donde hay cientos de miles de personas que provienen de la zona andina.

Este no es un problema privativo de esta zona sino que es un problema muy grande y es allí donde yo quisiera tocar un punto que Ud. indicó en su presentación y es que el desarrollo en que en [sic] todas partes entra la carretera, en todas partes entra la escuela, en todas partes entra las posibilidades de comercializar, el ambiente no es manejado por ellos, ya que ellos siempre y desde hace miles de años han tenido lazos de intercambios, de tráfico, de bienes, tremendo que movía cientos de miles de toneladas, pero que la gente lo hacía fuera del ámbito comercial, ya que el comercio no es la única manera de mover bienes ni personas.

Entonces se produce un choque cultural a la inversa, la gente que quiere ser efectiva, que quiere tomar acción en este ambiente es la persona que sufre muchas veces un choque cultural y no tanto el migrante, porque el migrante una vez que se establece no es la primera vez que lo hace sino que han habido muchas veces que ha venido antes por temporadas y cuando viene, ya tiene donde llegar. Yo se que en Arica la gente del interior tenía un lugar donde, llegar y cada una de las personas tenía esto, un lugar seguro donde llegar. No pasaba lo que sucede ahora que cuando uno viaja a otro lugar, tiene que empezar a buscar un hotel que más le convenga, estos señores no, ya que cuando venían tenían alojamiento seguro y propio en la ciudad, hasta cierto punto, era parte de un solo universo dentro del cual el hombre andino venía a resolver sus problemas. Antes de existir la ciudad, todos ellos tenían sus chacras acá abajo y todos ellos tenían el acceso a las caletas de donde provenía el huano, ya que si se piensa como se hacía la agricultura en la altura, necesariamente tenían que venir a buscar huano.

Es así que toda esta gente está aquí desde hace miles de años y no desde ayer solamente, esto me indica que hay un choque cultural, a la inversa.

Está el caso también que nosotros somos gente de buena voluntad y establecimos centros de salud, centros agronómicos, centro de escuelas y también 
llevamos un choque cultural muy violento. Este cambio no debe ser tan drástico, tan violento, tan amenazante, esta es una amenaza más de la realidad del cambio. Es este sentido de amenaza, de reto que recibe el hombre occidental, el hombre urbano, [muestra] no solamente con detalles que son diversos y que todos somos capaces de adaptarnos como ser a una película nueva, una camisa nueva, una comida, nueva, etc., sino que es toda una percepción y esto sí que es difícil soportarlo y esto todos los sufrimos ya que todos hemos viajado.

Sobre cualquiera de estos temas yo tengo experiencia. Por ejemplo, una cosa muy interesante es haber hecho que con la experiencia de Uds., gran parte de la agricultura andina sea eficiente, es una conclusión de todos los que tenemos experiencia en lo andino por lo que hemos visto en cualquier parte. El que se enfrenta con la agricultura andina descubre que ha sobrevivido por una excelente razón, que no sólo es eficiente sino que también la tierra es productiva

Ud tiene mucha razón cuando dice que hoy en día, en muchas partes, la productividad es baja, pero hay que ver también que esta baja productividad es el resultado progresivo del arrinconamiento, que la gente ha sido reducida a sus mínimos asentamientos, el asentamiento no puede ser más pequeño, cuando se llega a este mínimo, la próxima etapa es la que Ud. menciona y ahí también la gente empieza a emigrar, ya que dentro de esto hay una masa humana, hay una masa de conocimientos, de destrezas, que cuando ya no están las cosas apropiadas es mejor irse.

Esta es una de las zonas más ricas y cuando los... [sin transcripción] y europeos se enfrentaron y que muchos de ellos venían del Caribe y conocían las Indias, cuando vieron esta zona dijeron que era lo más rico que había. Darnos cuenta que uno puede a través de 3, 4 siglos perder poco a poco esta alta productividad, la que consistía en complementar continuamente la producción de alimentos con la producción de lana y combinarlo todo con lo que se tiene en la ciudad en una sola economía. Esta capacidad que tenían estos señores era fabulosa, ya que una cosa es manejar la provincia y otra cosa es manejar tanta serranía, tanto desierto, tanta precordillera, etc., lo que supone poder, lo que supone mando y estos señores tenían éste, pero también es importante tener en cuenta la experiencia gerencial, experiencia que les permitía planificar, coordinar todo esto.
Entonces sí la productividad es baja, hay que ver que esta baja productividad no es baja por ser tradicional andina, sino que es baja por la continua... [sin transcripción] y el último acto de esta larga historia es que las regiones... [sin transcripción] al insistirles que las regiones agrarias ocuparon gran parte de las riquezas complementarias de estos grupos humanos, al insistir que la familia tiene más de una casa y que caemos en más de un microclima, esto es propiedad ausentista.

Es muy natural para el hombre andino tener terrenos en diversos microclimas, y complementar lo que no se da en una zona con lo que se da en otra no a través del comercio sino que a través de la complementación de una serie de asentamientos humanos y ahora en cada zona los precios y medidas son distintos, no se habla de kilos, ni de litros, etc., sino lo que es apropiado para cada costumbre.

Es normal que el hombre andino tenga tres o cuatro casas, que es normal tener terrenos en varias partes distintas, que aún en el mismo terreno grande de toda la comunidad para alimentación, la tierra tiene que descansar. Sus informantes muchas veces le dicen que la tierra tiene que descansar 12 años y yo he visto en muchos lugares en que la tierra descansa 7 u 8 años. También hay muchos agrónomos y especialistas en que insisten que esto es atraso, que esto es baja productividad y como muchas veces nada entendemos, ya que muchos de nosotros somos ignorantes en esto, entonces hay que aprender a escuchar y comprender.

También está el caso de aquellos que van a cultivar la cebada, la compañía cervecera da la semilla y cuando viene la cosecha, la compañía garantiza el precio pero el agricultor insiste en que no y antes de cambiar prefiere venirse a la ciudad, mas fácil es convencerlo de venirse a la ciudad que venda sus productos, por la sencilla razón de que se pregunta ¿y yo qué como? y si se pregunta a la señora, también preguntará qué comerán los hijos. Entonces están convencido que la cebada no conviene pero los tubérculos sí.

Yo enseño en una Universidad Agraria y de hecho enseño en esta Universidad porque me interesa la agricultura andina. En esta Universidad hay muchos fitopatólogos que se ocupan de 1.000 plantas pero también hay algunos que se preocupan de tubérculos, y en el año 63 nuestra universidad descubrió que la amenaza más grande para los tubérculos andinos es un bicho llamado nematodo 
de oro el cual vive en la tierra y ataca las raíces de los tubérculos.

Nuestros expertos han descubierto que el ciclo vital del nematodo, desde que nace, se reproduce y pasa por todas sus etapas haciendo daños, es de 6 años. Esto nos demuestra la razón que tenía el hombre andino al pensar que la tierra debe descansar ese tiempo.

Sobre esto de las tierras me gustaría sugerir algo. Yo he visto en dos repúblicas distintas, con leyes de reforma distintas, lograda la reforma con antecedentes completamente distintos, pero todos coinciden en una cosa, cómo quitar las vallas, cómo quitar las tierras secundarias, terciarias de la población que se confunden con minifundios, que se confunde con ausentismo.

SR. SAAVEDRA: Respecto a la tenencia de la tierra, nosotros como Bienes Nacionales estamos dando titulo a los agricultores del altiplano en diversas, zonas y se les da un título por todos los predios que tienen, así sean 4, 5 o 10 predios chicos, minifundios. En este momento está paralizada la entrega de títulos por este problema de los predios chicos, ya que el deseo es que junten en uno solo. Esto se está estudiando con la gente del interior.

DR. MURRA: Permítame ser optimista y tomar la primera parte que señala, en el sentido que la Oficina está lista para dar títulos en diversos microclimas, lo que es un éxito. A continuación voy a explicar lo de la subdivisión para lo cual hay una excelente razón.

El agricultor obligatoriamente tiene que repartir el ingreso y el cuarto de hectárea que se tiene aquí contiene microclimas. Las condiciones naturales del surco de acá y las condiciones naturales del surco de allá no siempre son las mismas y también la tierra puede ser distinta.

Yo he leído algunos informes de la zona Pampa Algodonal, donde al repartirse ciertas tierras le tocaron a los Surire tierras más provechosas y diferentes a las tierras de los Camiña.

Dejando esto de lado, una parcela de 1 hectárea puede tener microclimas distintos en donde se van a dar surcos de tubérculos que se hielan y en otros surcos no. Estos hombres andinos debían tener una gran productividad y una enorme capacidad de almacenamiento.
A parte de que en cada parcela hay microclimas, está también lo segundo y es el hecho de que la tierra tiene que descansar, e ir rotando los cultivos.

Se da una explicación gráfica.

Lo que han hecho Uds. es una vieja tradición andina. ¿Hay días de comadres aquí?

SRA. NANCY ALANOCA: En la fecha de todos los Santos hay día de comadres y en este día se hacen muñecas de pan las cuales son intercambiadas entre las personas y eso encierra tanto la alegría como la pena que se entrega a la otra persona. Igual cosa sucede con la tierra, la cual se obtiene en el lugar mismo.

DR. MURRA: En los días de comadre, cada familia prepara comida, tragos, etc., y dan signos de hospitalidad y esperan horas para que venga alguien junto a las autoridades, cuando llegan dan vueltas al predio y constantemente están comiendo, tomando y mascando coca. Una vez que hacen el recorrido se sientan las señoras y los esposos se quedan de pié detrás de ellas. Y así lo hacen todas las familias.

SR. SAAVEDRA: Algo muy parecido se ve en los pueblos del interior. Cuando nos ha tocado realizar trabajos de topografía y se van avanzando kilómetros sin darnos cuenta, la gente llega justamente donde estamos a la hora de almuerzo llevando todo preparado. Es como si ellos supieran exactamente cuántos kilómetros vamos a recorrer y ahí están con el almuerzo como muestra de cariño.

SR. VALLEJO: Una de las cosas que sería interesante ver es poder regularizar la investigación a este tipo de situaciones. En primer lugar tener una claridad para poder orientar a las autoridades para no caer en los mismos errores. En segundo lugar, poder determinar algunas cosas concretas acerca de la propiedad andina, sobre la forma de adjudicarla y la forma de repartición.

En una visita realizada por nuestras autoridades de la zona del interior le manifestaron que el problema más grave que estaban teniendo con la legalización de tierra, era que esta legalización a través de la formula nuestra, estaba produciendo deformaciones y molestias contra la fórmula tradicional de repartición de la tierra. Toda la gente en el interior sabe cuál es concretamente su tierra y además hay un jefe que determina si es esa o no. 
De repente surge el problema que hay un tipo que es mas avispado y ve que hay una formula de hacerse de un pedazo de tierra a través de un título, el cual se obtiene con abogado y ante notario. Es así como sencillamente se consigue un título y al llegar arriba toda la comunidad lo rechaza.

El otro problema que teníamos y que estábamos tratando de conseguir información completa, es sobre la forma de repartición y de uso de la tierra en el altiplánico en este caso, en el caso concreto de los ganaderos.

DR. MURRA: Me alegro mucho de que ustedes estén conscientes de este problema y no creo que me necesiten a mí, que de hecho conocen mejor la situación, tienen más antecedentes y ahora sólo tienen que ver como se puede regularizar y adoptar una nueva política. Sobre todo que existe constancia en la necesidad de agricultura y que el agricultor siga produciendo.

Estoy muy de acuerdo que la investigación podría ayudar, pero gran parte de esta investigación no existe.

Cierta literatura andina si la leemos, sobre todo la de Ciro Alegría con su novela "El mundo es ancho y ajeno" es la historia real, ya que se las arreglo para quitar la tierra no a uno sino a muchos. Encuentro que el problema de los títulos es una cosa muy seria.

SR. SAAVEDRA: La legislación vigente es muy clara ya que la tierra se está dando a la gente que en realidad vive y está trabajando efectivamente la tierra.

DR. MURRA: Esta es una legislación excelente la cual no conocía. Hay que tomar en cuenta que no es de ahora que ellos están conviviendo con una población europea, sino que son siglos. Es por eso que digo lo importante que es el choque cultural, ellos se han adaptado, lo han manejado, han sobrevivido a pesar de todas las condiciones contrarias que han tenido que vivir.

Hay que insistir también en la otra parte, ya que no solo hay que defender sus derechos sino que hay que aprender...

Voy a contar una anécdota, tiempo atrás murió el jefe de hogar dejando a su mujer con un niño de meses los cuales se fueron a vivir a otro lado. Al cabo de 28 años regresó este joven pidiendo todo lo que le pertenecía a su padre. Nadie en el pueblo negó nada ya que de hecho le correspondía, pero también sabían que durante 28 años no había trabajado en la tierra y no había aportado nada a la comunidad. Esto lo reconocía también el joven, luego apareció una persona que dijo que su padre también había dejado deudas y que tenían que pagarse.

Este problema se solucionó y no hubo necesidad de tener un documento que lo justificara. Sino que todos sabían que era lo que correspondía y que estaba correcto.

Esta necesidad de ver la pluralidad de derechos, la... [sin transcripción] es un primer paso nada mas, lo que hay que ver es otra cosa. Hay una multiplicidad de derechos de acceso que diferentes seres humanos tienen derecho, no "privatividad", sino que derechos y comparten esos derechos con otros grupos humanos los cuales se pueden canjear.

Nuestro sistema de propiedad tiende a totalizar las cosas, la Municipalidad, el Estado siempre mantiene derechos, puede totalizar todo, igual cosa pasa con ellos, tienen múltiples acceso, esos derechos que para nosotros significa muestra conflictiva, para ellos no.

Todo esto nos lleva a la última conclusión que debemos aceptar la idea de que diversas cosas tienen diversas... [sin transcripción] como diversos tipos de football tienen diversos tipos de aficionados.

\section{SR. VALLEJO: Ofrece la palabra}

SR. DOUSSOULIN: En el transcurso de su exposición varias veces ha mencionado la Pampa Algodonal, lo cual me satisface mucho pues yo estuve a cargo de eso junto a Nancy Alanoca. Yo creo en la importancia que tiene ese proyecto y en ese sentido se le está dando el enfoque y una importancia real, en este momento. La importancia básica, mirándola desde el punto de vista estatal, es que ese sector se ha logrado con una inversión, se sabe que en el norte chileno para habilitar suelos las inversiones son de cualquier orden, sin un peso de gasto para el estado y se han habilitado alrededor de 100 hectáreas.

Pienso que esto es importante acotar a lo que ustedes, han señalado, en que no sólo basta el hombre andino de lo cual yo conozco muy poco pero el contacto que he tenido en este poco tiempo algo se aprende. El hombre andino baja con tal cantidad de conocimientos de todo tipo, pero como la realidad es diametralmente opuesta a la que se 
encuentra en el valle costero, especialmente en el valle de Azapa, encuentra limitantes muy serias en las estructuras de los suelos.

El error básico que encuentro es que al campesino se le ha dado mucho más de lo que ha necesitado y de lo que ha pedido. Recién me he estado dando cuenta del nuevo enfoque que hay que darle y ahí se puede llegar al concepto de verticalidad en el cual hay una integración de las economías andinas con esta explotación en el valle costero. Esa es la razón fundamental por la que no se ha pedido un peso al Estado.

Eso sí, entiendo yo, en absoluto se les puede dejar solos, sino que hay que hacerlo en la medida que ellos lo necesiten

DR. MURRA: La realidad es que no hay nadie que es andino nada más. ¿Porque vio la Pampa Algodonal?, porque había bajado tantas veces el valle de Azapa, etc.
Es importante lo que dice en el sentido que esto no ha costado al Estado, esto es un esfuerzo que hacen ellos. Este esfuerzo hay que respaldarlo, yo estoy completamente de acuerdo en que no hay que respaldarlo solamente por buena voluntad, sino que como estamos en crisis... ellos también cometerán muchos errores, ya que todos los cometemos continuamente, ya sea de juicio, de criterio, etc.

Ahora más, como decía recién, al perderse la herencia en grande y que permitía hacer de los diversos pisos una sola economía, es ficticio, estaba y ya no está y lo único que queda son fragmentos.

Hay que ayudarlos y también escucharlos para saber dónde quieren ayuda y cuándo.

SR. VALLEJO: Agradecemos mucho su presencia y sus palabras y esperamos tener la oportunidad de volver a reunirse para seguir tratando estos temas que son de interés.

\section{CARLOS SOLARI HERRERA}

Secretario
JORGE VALLEJO ARCO

Presidente 